\title{
Effect of crofelemer extract on severity and consistency of experimentally induced enterotoxigenic Escherichia coli diarrhea in newborn Holstein calves
}

\author{
A. G. V. Teixeira, ${ }^{*}$ L. Stephens, ${ }^{*}$ T. J. Divers, $†$ T. Stokol, ${ }^{*}$ and R. C. Bicalho ${ }^{* 1}$ \\ *Department of Population Medicine and Diagnostic Sciences, and \\ †Department of Clinical Sciences, College of Veterinary Medicine, Cornell University, Ithaca, NY 14853
}

\begin{abstract}
The objective of this study was to evaluate the effect of a standardized botanical extract of Croton lechleri, named crofelemer extract, on fecal dry matter and fecal scores on diarrheic newborn Holstein bull calves induced by enterotoxigenic Escherichia coli. A double-blinded randomized clinical trial was performed in which 60 newborn Holstein bull calves were clean caught and transported to an isolation facility where calves were individually housed and randomly assigned to 1 of 3 treatment groups: placebo (control), entericcoated formulation of crofelemer extract (ECROF), and nonenteric-coated formulation of crofelemer extract (CROF). Diarrhea was induced at first feeding with an inoculum of the enterotoxigenic Escherichia coli (ATCC 31616) administered with a third of the recommended dose of a colostrum replacer. All calves enrolled in this study received treatments starting on the second feeding (diarrhea onset) and treatments were administered before feeding time $(0600$ and $1600 \mathrm{~h})$ for 6 feedings consecutively. All calves in this study had failure of passive transfer. The only cause of death in this study was due to septicemia, accounting for 1 death out of each treatment group. All the calves were examined twice daily, within $2 \mathrm{~h}$ after feeding, from d 1 (prechallenge) until 10, on d 15, and a last examination on d 25 of life. Five parameters were evaluated during each examination; rectal temperature, clinical assessment of dehydration status, fecal scores, attitude, and appetite. No differences were observed between treatment groups for rectal temperature, attitude, and appetite. Fecal dry matter was analyzed as prechallenge fecal dry matter, dry matter during treatment, and fecal dry matter after treatment cessation. No difference in prechallenge fecal dry matter was observed and prechallenge fecal dry
\end{abstract}

Received February 27, 2015.

Accepted June 28, 2015.

${ }^{1}$ Corresponding author: rcb28@cornell.edu matter was used as a covariate in the models. Fecal dry matter during treatment was significantly higher for ECROF calves when compared with control calves and CROF calves. Additionally, ECROF fecal dry matter after treatment cessation had a statistical tendency to be higher when compared with control calves. Together, these results suggest that enteric-coated formulation of the standardized crofelemer extract, a natural-product with antisecretory properties, can significantly increase fecal dry matter of neonatal calves with experimentally induced enterotoxigenic Escherichia coli diarrhea. More research is needed to test the efficacy of enteric-coated crofelemer on incidence and severity of secretory diarrhea on calves under natural challenge conditions.

Key words: calves, diarrhea, fecal dry matter, crofelemer

\section{INTRODUCTION}

Considerable economic losses may be incurred from neonatal diseases during calf rearing. In 2010, diarrhea $(18 \%)$ and pneumonia (16\%) were the most common disorders affecting preweaned heifers reported by the National Animal Health Monitoring System (USDA, 2011). Diarrhea is a multifactorial disease that can be caused by infectious and noninfectious factors (Walker et al., 1998; O'Handley et al., 1999). The risk factors known to be associated with diarrhea are management (environmental condition), nutritional state, immune status, and pathogen exposure (Klein-Jöbstl et al., 2014; Al Mawly et al., 2015). Enteropathogens, such as viruses, bacterial, and protozoa, are often identified as etiological agents in calf diarrhea. The most common enteropathogens described in the literature include Escherichia coli, Salmonella spp., Cryptosporidium, and rotaviruses (Moon et al., 1978; O'Handley et al., 1999; Gulliksen et al., 2009; da Silva Medeiros et al., 2015).

Enterotoxigenic E. coli and neonatal diarrhea have been extensively studied in the last 4 decades (Bywater and Logan, 1974; Radostits, 1975; Acres, 1985). Much has been done to prevent early life contamination with enteropathogens: minimal dam-calf contact, colostrum 
pasteurization, and disease-control practices (Moon and Bunn, 1993; Godden, 2008; Naylor, 2009). In spite of efforts controlling neonatal diarrhea, it still remains as a major concern in the beef and dairy industry, with serious effects on animal welfare and profitability (USDA, 2011; Hughes, 2013).

Many important aspects of prevention and treatment of diarrhea relate to Escherichia coli. Vaccination of the cows before parturition can effectively provide antibodies against strains of enterotoxigenic Escherichia coli (ETEC; Frantz et al., 1987); the vaccination can also prevent neonatal diarrhea (Nagy, 1980). Antibiotics have been used as treatment against diarrhea caused by Escherichia coli (Sunderland et al., 2003); however, the use of antibiotics is under scrutiny due to concerns of bacterial resistance that could affect human health. Alternative therapeutic treatments for diarrhea have become more common. Studies have been conducted testing the efficacy of organic and inorganic zinc, probiotics, and even bacteriophages targeting enteropathogens for treatment of diarrhea in calves (Muscato et al., 2002; Bicalho et al., 2012; Glover et al., 2013).

The Food and Drug Administration recently approved crofelemer, a polyphenolic molecule isolated from the latex of the plant species Croton lechleri of the family Euphorbiaceae indicated for idiopathic human immunodeficiency virus (HIV)-associated secretory diarrhea. Crofelemer has been studied for its antisecretory actions that involve the inhibition of 2 distinct chloride channels on the luminal membrane of the intestine: chloride fibrosis transmembrane conductance regulator (CFTR) and calcium-activated chloride channel (Tradtrantip et al., 2010). The CFTR is expressed at the apical membrane of enterocytes and plays an important role in the intestinal physiology. Enterotoxigenic Escherichia coli secretes bacterial toxins that leads to the activation of CFTR and calcium-activated chloride channel, consequently leading to chloride secretion and intestinal fluid hyper secretion (Thiagarajah and Verkman, 2013).

To the best of our knowledge, the efficacy of crofelemer extract as an antidiarrheal drug has not been previously evaluated in a neonatal bovine secretory diarrhea model. Here, we investigate the antisecretory potential of crofelemer extract in newborn Holstein bull calves challenged with ETEC in the first day of life. The objective of our study was to evaluate the effect of 2 formulations of crofelemer extract on diarrhea severity and consistency of experimentally induced diarrhea in newborn Holstein calves in the first $5 \mathrm{~d}$ of life. As a second objective, the overall health status and performance of the calves were evaluated until $25 \mathrm{~d}$ of life.

\section{MATERIALS AND METHODS}

\section{Experimental Design}

This experimental protocol was approved by the Institutional Animal Care and Use Committee of Cornell University (Protocol number 2013-0075). Sample size was based on a fecal DM measured on a pilot study. Using a mean difference between groups of 1.4, a treatment group standard deviation of 1.2 and a placebo group standard deviation of 1.7 , with a treatment group ratio of 1 , assuming a desired type I error rate of $5 \%$, and a power of $80 \%$, a sample size of 18 calves per group was calculated. As $10 \%$ of mortality was anticipated, a total of 20 calves were enrolled in the study.

The study design was a double-blinded randomized clinical trial. Randomization was performed a priori to beginning of the trial using Excel (Microsoft Corp., Redmond, WA) random function to create a balanced number of calves per treatment group $(\mathrm{n}=20$ calves per group). Data collection blindness was simplified by treatment tablet similarities; tablets were manufactured to have same appearance (same color and size) and were inodorous. A total of 60 Holstein bull calves from one commercial dairy farm (King Ferry, NY) were enrolled in the study. Calves were clean caught at parturition (minimizing animal contact between dam and calf or calf and maternity bed) and transported within $2 \mathrm{~h}$ to an isolation facility for research animals at Cornell University. All the calves were enrolled in the study within $3 \mathrm{~h}$ after birth. Transportation and vehicle-cleaning procedure were performed accordingly to the Animal Care and Use Procedure. Briefly, calves were transported using an adapted cargo van; calves were kept inside individual crates with proper ventilation and transported directly from the farm to the research facility. Cleaning and sanitization of the vehicle was performed 30 min before and immediately after transportation.

Calves were individually housed in $16-\mathrm{m}^{2}$ rooms with controlled temperature and humidity. Each isolation room had an individual inner-room containing all the necessary instrumentation for feeding, treatments, weighing, cleaning, and data collection. No equipment was shared between calves. Additionally, calves were unable to have any contact with other calves or outside areas. Cleaning and sanitation of bottles, nipples, and buckets used to hold milk (before and after each feeding), water (once daily), and calf starter (twice weekly) were manually performed by a 3 -step cleaning procedure. The 3 -step cleaning procedure consisted of rinsing all the equipment with lukewarm water, scrubbing a 
mixture of hot water and alkaline detergent solution, and finally rinsing in chlorinated water.

Calves were fed antibiotic-free milk replacer (Nutrablend 22/20, Ranch-Way, Fort Collins, CO) by bottle on a $10 \% \mathrm{BW}$ basis twice a day (0600 and $1800 \mathrm{~h}$ ) during the first $3 \mathrm{~d}$ of life. Calves were gradually removed from bottles and encouraged to drink from the bucket. Water was available ad libitum from d 1 until the end of the study. All calves were kept in the study until 25 $\mathrm{d}$ of life with ad libitum access to calf starter $(18 \% \mathrm{CP}$, DuMOR, Patterson, NY) starting on the seventh day of life.

\section{Escherichia coli Inoculum and Challenge}

All the calves were challenged using an enterotoxigenic Escherichia coli (ETEC) serotype O9:K35:K99 (ATCC 31616). The ETEC inoculum used in our study was prepared 2 wk previously to the beginning of the trial. Standard ETEC ATCC bacteria activation was performed using trypticase soy broth (Trypticase, Becton, Dickinson and Co., Franklin Lakes, NJ) to grow the bacteria for $8 \mathrm{~h}$ and then on Luria-Bertani agar (Difco LB Agar, Becton, Dickinson and Co.) for $18 \mathrm{~h}$ at $37^{\circ} \mathrm{C}$. The bacteria was suspended in PBS with $10 \%$ dimethyl sulfoxide and stored in $10-\mathrm{mL}$ aliquots at $-70^{\circ} \mathrm{C}$. The mean inoculum titer was $4 \times 10^{10} \mathrm{cfu} / 10 \mathrm{~mL}$.

All the calves were challenged at the research facility within $6 \mathrm{~h}$ of life. A 1-L mixture of freshly prepared colostrum replacer containing $157 \mathrm{~g}$ of colostrum-replacer powder and approximately $35 \mathrm{~g}$ of IgG (Calf Colostrum Replacer, Land O Lakes, Arden Hills, MN) was mixed with $10 \mathrm{~mL}$ of thawed ETEC inoculum. The mixture was administered within $1 \mathrm{~min}$ after preparation for each calf via esophageal feeder. Feeders were used only once per calf and properly discarded (Oral Calf Feeder, Jorvet, Loveland, CO).

\section{Treatment Administration and Data Collection}

Calves were assigned into 1 of 3 treatment groups: control (CTR; $\mathrm{n}=20)$, enteric-coated formulation of crofelemer extract (ECROF; $\mathrm{n}=20$ ), and nonentericcoated formulation of crofelemer extract (CROF; n $=20$ ). The treatments were administered before each meal for $3 \mathrm{~d}$ consecutively (total of 6 treatments per calf) starting on the first feeding after challenge.

Only 1 trained caretaker fed the animals during the whole study period, and only 1 member of the study group was responsible for the challenge, treatment administration, and data collection. Calves were weighed at birth and 10, 15, and $25 \mathrm{~d}$ of life using a portable scale. Blood samples were collected via jugular venipuncture using an 18-gauge by $3.8-\mathrm{cm}$ needle in an 8-mL vacuum tube (Becton, Dickinson and Co.) without anticoagulant for serum. Blood samples were collected between the morning and afternoon feedings; the first blood sample (baseline) was taken immediately before challenge and 4 subsequent blood samples were taken daily.

Serum was harvested following centrifugation at $2,000 \times g$ for 15 min at $4^{\circ} \mathrm{C}$. Serum IgG was measured using the second blood collection (second day of life) using a radial immunodiffusion assay according to kit instructions (Bethyl Laboratories Inc., Montgomery, TX). Total solids were evaluated in serum samples using an optical refractometer.

All the calves were examined twice daily, within $2 \mathrm{~h}$ after feeding, from d 1 (prechallenge) to 10 , on d 15 , and a last examination on d 25 of life. Five parameters were evaluated during each examination: rectal temperature, dehydration status, fecal scores, attitude, and appetite.

Dehydration status, attitude, and appetite scores were based on a numerical scale as follows. Dehydration status ranged from $0=$ normal, eyes are bright and skin feels pliable; 1 = mild dehydration, slight loss of skin elasticity, skin tent $<3 \mathrm{~s}$, eyes not recessed into orbit; $2=$ moderate dehydration, skin tent $>3 \mathrm{~s}$ but $<10 \mathrm{~s}$, eyes slightly recessed into orbit; to $3=$ severe dehydration, skin tent $>10 \mathrm{~s}$, eyes markedly recessed into orbit. Attitude ranged from $0=$ alert, $1=$ depressed, to $2=$ non-responsive. Appetite ranged from $0=$ normal, $1=$ consuming less than half of the meal, $2=$ consuming less than $25 \%$ of the meal, to $3=$ not consuming. Fecal scores were a 5 -point scale (Figure 1) based on fecal consistency. Fecal samples were also collected (20-mL plastic vials) during each clinical evaluation via digital stimulation on the calf's rectum to evaluate percentage of DM of the feces. Fecal DM was determined as described by Bellosa et al., (2011). Briefly, 5 to $20 \mathrm{~g}$ of the sample was weighed on a precision digital scale (6202-1S, Sartorius, Elk Grove, IL) and then dried at $108^{\circ} \mathrm{C}$ for $24 \mathrm{~h}$ in an oven (model 10 Lab Oven, Quincy Lab, Chicago, IL) and reweighed immediately to determine the percent DM.

\section{Fluid Therapy}

As a rescue treatment, an oral electrolyte (Hydralyte, Lloyd Inc., Shenandoah, IA) was fed via bottle as an extra meal administered in between meal hours to calves that had a fecal score $\geq 3$ and dehydration score $\geq 2$. Intravenous fluid therapy using isotonic sodium bicarbonate was administered by jugular vein catheter (70 mL/kg per hour) if calves were unable to stand, presenting very weak or no suckle reflex, and dehydration score $\geq 2$. 


\section{Statistical Analyses}

A total of 24 examinations or samples were collected per calf in our study. For the continuous data collected on fecal DM and rectal temperature, calf samples were used as prechallenge (defined as the first examination or sample before the challenge), during treatment days (defined as the calf average data collected during $3 \mathrm{~d}$ of treatments; second to the seventh examination or sample), and after treatment cessation (defined as the calf average data collected after treatment cessation; eighth to the 24th examination or sample). Data on fecal DM and temperature was averaged within calf.

Statistical analyses were performed using JMP 10 (SAS Institute Inc., Cary, NC). An ANOVA was used to evaluate serum IgG. Blood samples from the second collection (postchallenge) were used to measure serum IgG. Intraassay and interassay coefficients of variation for IgG were 3.2 and $3.6 \%$, respectively.

To evaluate the effect of treatment on the dichotomized clinical outcomes - diarrhea (fecal score $\geq 3$ ), depression (attitude score $\geq 1$ ), hypophagia (appetite score $\geq 1$ ), dehydration (dehydration score $\geq 2$ ), and oral electrolyte administration (yes or no)-Fisher's exact test was used to compare the percentage of calves affected in each treatment group against control calves. Additionally, the number of events (diarrhea, depression, hypophagia, dehydration, and oral electrolyte administration) recorded during treatments days and after treatment cessation was evaluated using ANOVA comparing the treatment groups against the control group.

A mixed general linear (MGL) model was used to analyze the effect of treatment on rectal temperatures.
Initial rectal temperature (prechallenge) was used as a covariate in the MGL models to evaluate rectal temperature. The MGL models were used to analyze the effect of treatment on ADG. Calves enrolled in the ECROF group had a numerically smaller average birth weight at enrollment when compare with calves on CTR and CROF groups $(P=0.14)$; for that reason, birth weight was used as a covariate in the MGL models to evaluate ADG. Average daily weight gain was calculated on d 10,15 , and 25 of the calves' life by subtracting the birth weight from the weight at 10,15 , and $25 \mathrm{~d}$ of life; these variables were then used as the outcome variable for the MGL models.

A similar MGL model was used to analyze the effect of treatment on serum TS. The data on TS was measured at prechallenge (d 0) and d 1, 2, 3, and 4 of calves' life. Prechallenge TS measurement was used as covariate in this model. To control for repeated measures, the animal identification number was added in the model as a random effect.

For all general linear mixed models, the assumption that the residuals were normally distributed was assessed by visually evaluating the distribution plot of the studentized residuals. Statistical significance was declared when $P \leq 0.05$ and statistical tendencies were declare when $0.05<P \leq 0.10$. Results are presented as least squares means and standard error of the mean.

\section{RESULTS}

Three calves, one in each group, were euthanized within $72 \mathrm{~h}$ of life due to signs of bacteremia. Euthanasia (penetrating captive bolt) was carried out by trained personnel. All data collected from these calves were not

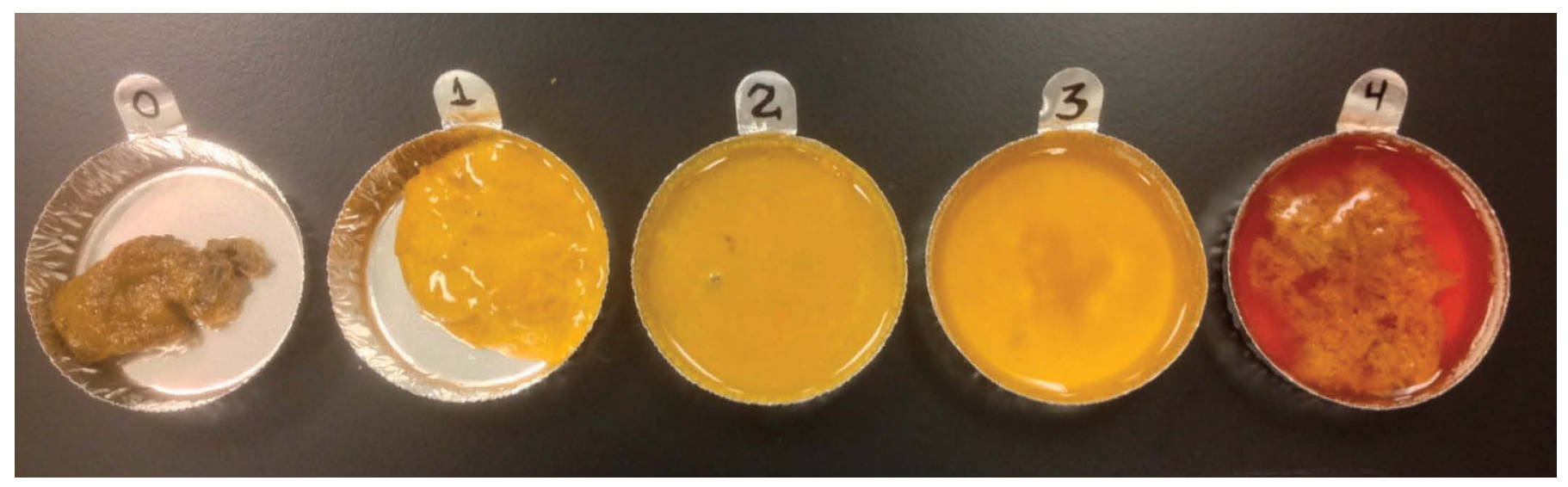

Figure 1. Fecal scores were based on diarrhea severity and the picture represents the 5-point scale used to visually assess calf diarrhea on milk fed calves. Another 4 samples were collected on d 15 and 25 of calves' life. From the left to the right; $0=$ formed feces with normal color, $1=$ pasty with normal color, $2=$ liquid with normal color, $3=$ watery with normal color, and $4=$ watery with abnormal color. Color version available online. 
used in the analysis. Those calves were the only calves in the present study that needed i.v. fluid therapy and as a result, no i.v. data were used. All euthanized calves were submitted to the Animal Health Diagnostic Center (Cornell University) for a postmortem examination and sepsis was confirmed.

No statistical differences were observed for 24-h serum IgG $(\mathrm{mg} / \mathrm{mL})$ between treatment groups; 2.80 $( \pm 0.29), 3.00( \pm 0.28)$, and $2.85( \pm 0.27)$ for CTR, ECROF, and CROF groups, respectively $(P=0.87)$. Prechallenged fecal DM (Figure 2) was not significantly different between treatment groups: 21.41 ( \pm 1.63$)$, $20.67( \pm 1.55)$, and $21.25 \%( \pm 1.64)$ for CTR, ECRO, and CROF, respectively $(P=0.96)$. Average fecal DM was significantly higher for ECROF $(15.45 \pm 1.55 \%)$ calves during treatment days when compared with CTR calves $(11.15 \pm 1.63 \%)$ and CROF calves (11.16 $\pm 1.64 \%)$; however, no difference was observed between CROF and CTR calves $(P=0.96)$. The ECROF calves had a statistical tendency to have higher average fecal DM when compared with control calves $(P=0.08)$ following cessation of treatment. However, no significant difference in fecal DM after treatment cessation was observed between CROF and ECROF $(P=0.73)$ nor CROF and CTR $(P=0.16)$.

Serum harvested from 5 blood samples per calf were used to measure TS (Figure 3) using an optical refractometer. There were no baseline (d 0) differences in TS between treatment groups $(P=0.54)$. A statistical tendency was noted on d 1 collection for serum TS to be lower for calves in the ECROF group when compared with the CTR group $(P=0.06)$. No differences were found between treatment groups on d 2 . Serum TS were significantly higher for CTR calves when compared with ECROF calves $(P=0.03)$ and when compared with CROF calves $(P=0.05)$ on d 3 , but no differences were observed between ECROF and CROF groups. Additionally, serum TS were significantly higher on d 4 collection for calves in CTR group when compared with ECROF $(P=0.05)$ and CROF $(P=0.02)$.

Diarrhea incidence prechallenge was similar between treatment groups, at $5.6 \%$ for CTR, ECROF, and CROF $(P=1)$. During treatment days, calves in the control group had $68.4 \%$ diarrhea incidence, whereas

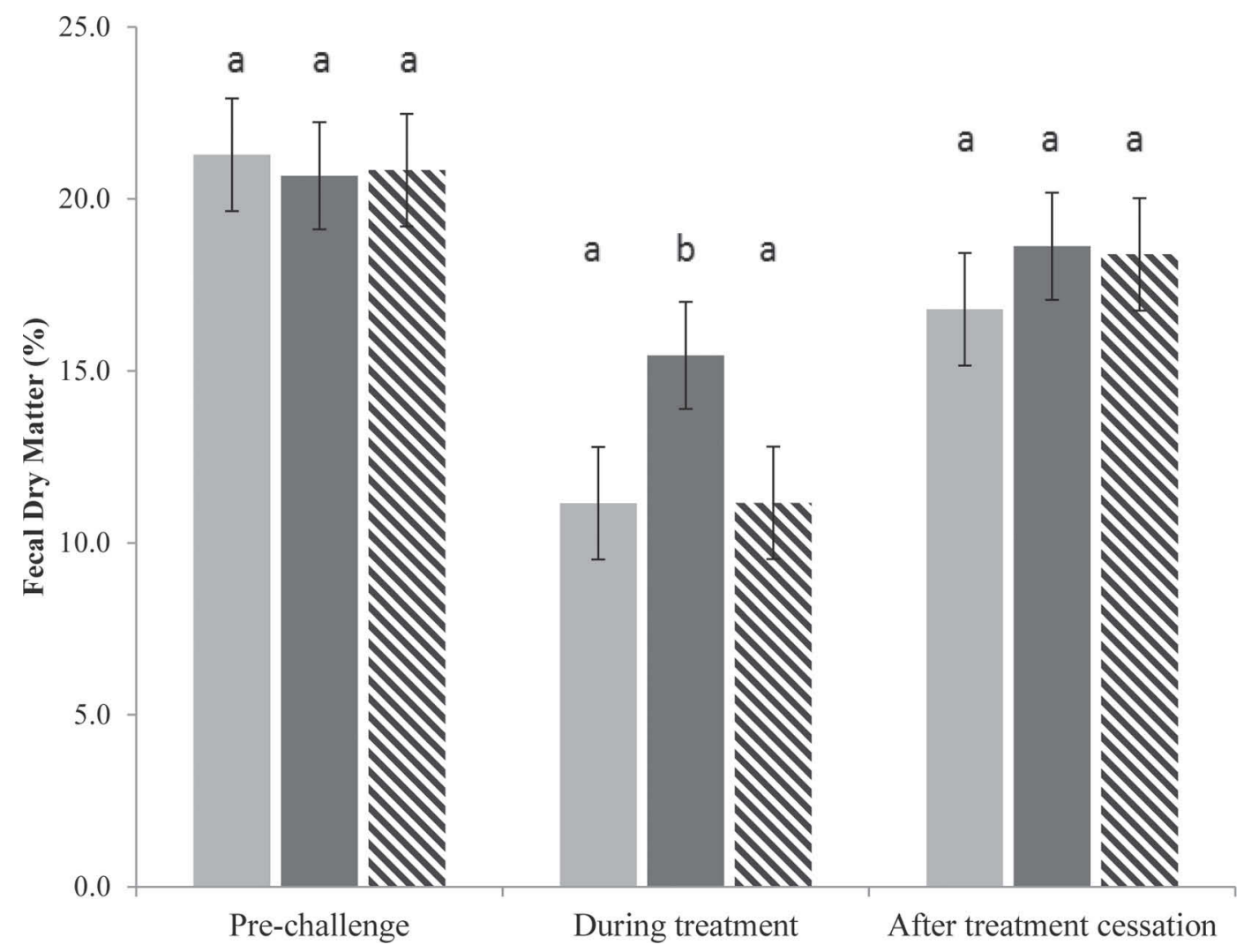

Figure 2. Effect of treatment on calves' fecal DM content (percentage of DM) measured on prechallenge, during treatment days, and after treatment cessation. The light gray bar represents control, the dark gray bar represents enteric-coated group, and the diagonal striped bar represents nonenteric-coated group. Data are presented as means and SEM. Different letters (a,b) within period (prechallenge, during treatment days, and after treatment cessation) represent statistical differences $(P<0.05)$. 


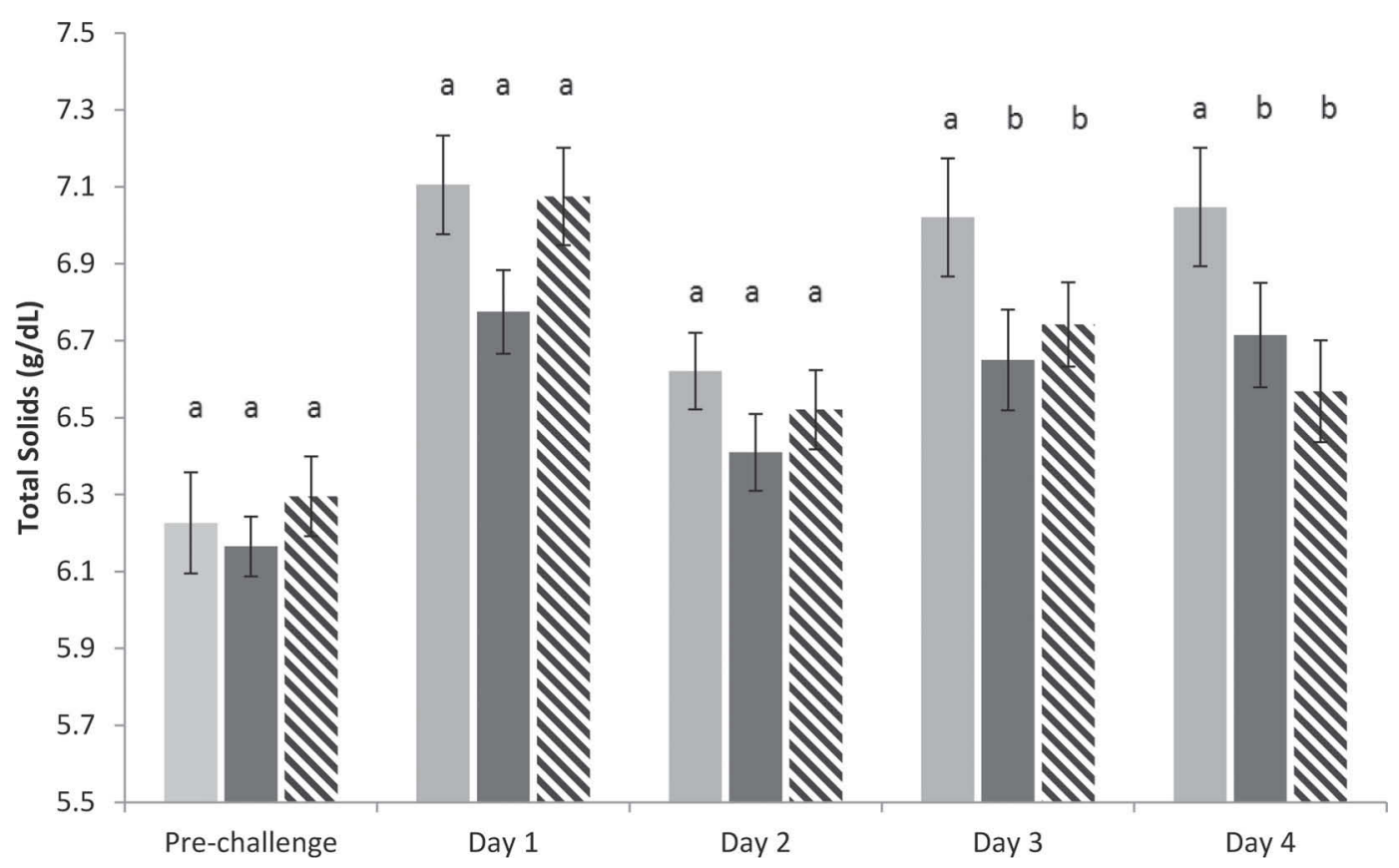

Figure 3. Effect of treatment on calves' serum TS (g/dL) measured on blood samples collected 5 times at prechallenge (d 0), d 1, 2, 3, and 4 of life. The light gray bar represents control, dark gray bar represents enteric-coated group, and diagonal striped bar represents nonentericcoated group. Data are presented as means and SEM. Different letters (a,b) within period (prechallenge, d 1, 2, 3, and 4) represent statistical differences $(P<0.05)$.

calves in the CROF group had $84.2 \%(P=0.44)$ and calves in the ECROF group had $57.9 \%(P=0.74)$. Additionally, no differences were found between the mean duration of diarrhea between CTR, CROF, and ECROF. However, after treatment cessation, CTR calves had $57.9 \%$ incidence of diarrhea whereas only $15.8 \%$ of the calves in the ECROF group were diarrheic $(P$ $=0.007)$; no differences were found between the CTR group and calves in the CROF group. Moreover, diarrhea duration was significantly lower for calves in the ECROF group when compared with calves in the CTR group $(P=0.02)$.

The percentage of calves with decreased appetite (hypophagia), and dehydration were not found to be significantly different between treatment groups at the prechallenge, during treatment days, or after treatment cessation between treatment groups (Table 1). In addition to the clinical evaluation, rectal temperature was not significantly different between treatment groups before challenge, at $38.9( \pm 0.07), 38.8( \pm 0.07)$, and $38.9^{\circ} \mathrm{C}$ $( \pm 0.07)$ for CTR, ECROF, and CROF, respectively. No differences were found between treatment groups for calves' rectal temperature during treatment days $(P=$ $0.90)$ or after treatment $(P=0.94)$.

Calf birth weight $(\mathrm{kg})$ was not significantly different between treatment groups, at $42.7( \pm 1.13), 42.4$ $( \pm 1.13)$, and $40.2( \pm 1.10)$ for CTR, ECROF, and
CROF, respectively $(P=0.14$; Table 2$)$. At $10 \mathrm{~d}$ of life, a statistical tendency was observed, average daily gain was $177.4( \pm 43.9), 285.4( \pm 41.7)$, and $223.5 \mathrm{~g} / \mathrm{d}$ $( \pm 43.9)$ for CTR, ECROF, and CROF groups, respectively $(P=0.08)$. No statistical difference was observed between treatment groups at $15 \mathrm{~d}$ of life, ADG was $233.9( \pm 47.9), 292.9( \pm 42.9)$, and $254.0 \mathrm{~g} / \mathrm{d}( \pm 46.5)$ for CTR, ECROF, and CROF groups, respectively ( $P$ $=0.34)$. At $25 \mathrm{~d}$ of life, ADG was $219.2( \pm 47.9), 281.2$ $( \pm 44.1)$, and $257.4 \mathrm{~g} / \mathrm{d}( \pm 46.5)$ for CTR, ECROF, and CROF groups, respectively $(P=0.43)$.

\section{DISCUSSION}

In the present study, 2 formulations of crofelemer extract were evaluated; an enteric-coated (ECROF) and a noncoated formulation (CROF). The ECROF formulation was significantly more effective in increasing fecal DM during treatment days when compared with CROF and placebo. Crofelemer has been studied for its antisecretory properties; crofelemer molecules can inhibit active chloride channels of enterocytes (Tradtrantip et al., 2010). Crofelemer targets the enterocyte extracellular surface and, as such, an additional challenge is faced as the substance is susceptible to be washed away once in the lumen of the intestine due secreted fluid (Thiagarajah and Verkman, 2003). Tradtrantip et al. 
(2010) reported in vitro results indicating that crofelemer resisted washout, with less than $50 \%$ reversal of CFTR inhibition after $4 \mathrm{~h}$.

It is important to highlight that the effect reported by our study, favoring the enteric-coated form and not the nonenteric-coated form, could be attributed to the enteric-coating process during the tablet formulation. Constable et al. (2005) reported that average abomasal $\mathrm{pH}$ was 3.22 during a 24 -h interval on calves fed all milk-protein milk replacer twice daily, and that the minimum preprandial $\mathrm{pH}$ was 1.34 and maximum postprandial $\mathrm{pH}$ was 6.07 . The protection from the acidity of the abomasum by the enteric-coating process could have led to a higher concentration of the active component of the treatment into the intestinal lumen.

Fecal scores were used to visually assess calves affected with diarrhea. No differences were observed between treatment groups for the period of treatment. However, after treatment cessation, calves in the enteric-coated group had lower incidence and lower number of diarrhea events. This finding is consistent with the data regarding oral electrolytes. Calves in the enteric-coated group had a tendency to received lower oral fluid therapy during treatment days and after treatment cessation.

In the current study, secretory diarrhea was induced by ETEC, which can rapidly lead to a high state of dehydration and life-threatening conditions for newborn animals. As described by Hartmann et al. (1984) and Hartmann and Reder (1995), calves with watery diarrhea can lose up to $21 \%$ of its BW. However, in the present study, no differences were found between treatment groups when evaluating dehydration during days of treatment. Nevertheless, diarrheic calves with suckling reflexes and moderate dehydration were given oral fluid therapy. It could be possible that the quick detection of dehydration and subsequent fluid therapy (oral electrolyte administration) prevented the induced secretory diarrhea from causing severe dehydration.

Serum TS were used as a laboratory measurement of dehydration. In cases of secretory diarrhea, water moves from the circulatory system into the intestinal lumen, concentrating the solid components of blood, leading to a higher TS concentration and higher reading by the optical refractometer. In the present study, control calves had a strong statistical tendency to have higher TS readings during second day of treatment when compared with enteric-coated formulation of crofelemer extract treated calves. Moreover, after treatment cessation, control calves had higher serum TS reading when compared with crofelemer-treated calves. Laboratory evaluation of blood components can be used to measure dehydration status by the analyses of TS, packed cell volume, creatinine, and urea. However, considerable variation on blood components could be observed when 
Table 2. Calf birth weight $(\mathrm{kg})$ and the effect of treatment on ADG $(\mathrm{g} / \mathrm{d})$ at 10,15 , and $25 \mathrm{~d}$ of life

\begin{tabular}{|c|c|c|c|c|}
\hline \multirow[b]{2}{*}{ Item } & \multicolumn{3}{|c|}{ Treatment group ${ }^{2}$} & \multirow[b]{2}{*}{$P$-value } \\
\hline & CTR & ECROF & $\mathrm{CROF}$ & \\
\hline Birth weight $(\mathrm{kg})$ & $42.7(1.13)$ & $42.4(1.13)$ & $40.2(1.10)$ & 0.14 \\
\hline $10-\mathrm{d} \mathrm{ADG}^{3}$ & $177.4(43.9)$ & $285.4(41.7)$ & $223.5(43.9)$ & 0.08 \\
\hline $15-\mathrm{d}$ ADG & $233.9(47.9)$ & $292.9(42.9)$ & $254.0(46.5)$ & 0.34 \\
\hline 25-d ADG & $219.2(47.9)$ & $281.2(44.1)$ & $257.4(46.5)$ & 0.43 \\
\hline
\end{tabular}

supportive therapy is in place (Abutarbush and Petrie, 2007). Constable et al. (1998) reported that these parameters are not as good parameters to determine dehydration as degree of enophthalmos and total protein measurements.

All calves enrolled in our trial had failure of passive transfer. This was expected given the intentional reduced amount of colostrum replacer administered to the calves during the challenge. In our study, septicemia was expected in some calves due to the nature of the challenge; calves were given a high amount of bacteria with a low amount of colostrum. Neonatal calves are highly susceptible to septicemia because they are dependent on colostral immunoglobulins (Fecteau et al., 2009). However, we experienced a lower mortality rate and only 1 calf in each group was euthanized due septicemia.

Hyperthermia and hypothermia were not found to be significantly different between treatment groups in this trial at prechallenge, during treatment days, and after treatment cessation (data not shown). Lower temperatures were found elsewhere to be significantly associated with hypovolemic shock in cases of severe dehydration (Sunderland et al., 2003; Nagy and Fekete, 2005).

Average daily gain was not significantly different between treatment groups. Calves in the ECROF group had a statistical tendency to gain $108 \mathrm{~g} / \mathrm{d}$ more when compared with control group during the first $10 \mathrm{~d}$ of life. Newborn calves are more susceptible to fluid losses due to their higher total water content and higher extracellular fluid volume (Hartmann et al., 1984; Hartmann and Reder, 1995). It is possible that the effect observed on weight gain difference was due to water losses in the feces. A study conducted in the early 1970s comparing nondiarrheic calves with calves affected with spontaneous diarrhea reported that $96 \%$ of the weight lost in a diarrheic calf is due to water loss, in which $71.4,16.1$, and $12.5 \%$ of water loss was due fecal, urinary, and insensible, respectively (Phillips et al., 1971). However, due the lack of calf starter consumption data, little can be concluded from the data presented for the ADG calculated for the entire study period.

\section{CONCLUSIONS}

Newborn diarrhea was induced using ETEC. Diarrheic calves were treated with 2 forms of crofelemer extract (enteric- and nonenteric-coated) administered twice a day for $3 \mathrm{~d}$ consecutively and compared with control calves (placebo). Results herein presented demonstrated that enteric-coated crofelemer extract, a natural product with antisecretory properties, can significantly increase fecal DM on diarrheic neonatal calves. More research is needed to test the efficacy of enteric-coated crofelemer on incidence and severity of secretory diarrhea on diarrheic calves under natural challenge conditions.

\section{REFERENCES}

Abutarbush, S. M., and L. Petrie. 2007. Treatment of hypernatremia in neonatal calves with diarrhea. Can. Vet. J. 48:184-187.

Acres, S. D. 1985. Enterotoxigenic Escherichia coli infections in newborn calves: A review. J. Dairy Sci. 68:229-256.

Al Mawly, J., A. Grinberg, D. Prattley, J. Moffat, J. Marshall, and N. French. 2015. Risk factors for neonatal calf diarrhoea and enteropathogen shedding in New Zealand dairy farms. Vet. J. 203:155160.

Bellosa, M. L., D. V. Nydam, J. L. Liotta, J. A. Zambriski, T. C. Linden, and D. D. Bowman. 2011. A comparison of fecal percent dry matter and number of cryptosporidium parvum oocysts shed to observational fecal consistency scoring in dairy calves. J. Parasitol. 97:349-351.

Bicalho, M. L. S., V. S. Machado, D. V. Nydam, T. M. A. Santos, and R. C. Bicalho. 2012. Evaluation of oral administration of bacteriophages to neonatal calves: Phage survival and impact on fecal Escherichia coli. Livest. Sci. 144:294-299.

Bywater, R. J., and E. F. Logan. 1974. The site and characteristics of intestinal water and electrolyte loss in Escherichia coli-Induced diarrhoea in calves. J. Comp. Pathol. 84:599-610.

Constable, P. D., A. F. Ahmed, and N. A. Misk. 2005. Effect of suckling cow's milk or milk replacer on abomasal luminal $\mathrm{pH}$ in dairy calves. J. Vet. Intern. Med. 19:97-102.

Constable, P. D., P. G. Walker, D. E. Morin, and J. H. Foreman. 1998 Clinical and laboratory assessment of hydration status of neonatal calves with diarrhea. J. Am. Vet. Med. Assoc. 212:991-996.

da Silva Medeiros, T. N., E. Lorenzetti, A. F. Alfieri, and A. A. Alfieri. 2015. Phylogenetic analysis of a G6P[5] bovine rotavirus 
strain isolated in a neonatal diarrhea outbreak in a beef cattle herd vaccinated with G6P[1] and G10P[11] genotypes. Arch. Virol. 160:447-451.

Fecteau, G., B. P. Smith, and L. W. George. 2009. Septicemia and meningitis in the newborn calf. Vet. Clin. North Am. Food Anim. Pract. 25:195-208.

Frantz, J. C., P. K. Bhatnagar, A. L. Brown, L. K. Garrett, and J. L. Hughes. 1987. Investigation of synthetic Escherichia coli heatstable enterotoxin as an immunogen for swine and cattle. Infect. Immun. 55:1077-1084.

Glover, A. D., B. Puschner, H. A. Rossow, T. W. Lehenbauer, J. D. Champagne, P. C. Blanchard, and S. S. Aly. 2013. A double-blind block randomized clinical trial on the effect of zinc as a treatment for diarrhea in neonatal Holstein calves under natural challenge conditions. Prev. Vet. Med. 112:338-347.

Godden, S. 2008. Colostrum management for dairy calves. Vet. Clin. North Am. Food Anim. Pract. 24:19-39.

Gulliksen, S. M., E. Jor, K. I. Lie, I. S. Hamnes, T. Loken, J. Akerstedt, and O. Osteras. 2009. Enteropathogens and risk factors for diarrhea in Norwegian dairy calves. J. Dairy Sci. 92:5057-5066.

Hartmann, H., L. Finsterbusch, and R. Lesche. 1984. Fluid balance in calves. 2. Body fluid compartments depending on the age of the animal and changes caused by diarrhea. Arch. Exp. Veterinarmed. 38:913-922.

Hartmann, H., and S. Reder. 1995. Effects of dehydration on functional parameters of fluid balance as well as effectiveness of rehydration using crystalline or colloidal infusion drips in calves. Tierarztl. Prax. 23:342-350.

Hughes, H. 2013. Raised replacement heifers: Some economic considerations. Vet. Clin. North Am. Food Anim. Pract. 29:643-652.

Klein-Jöbstl, D., M. Iwersen, and M. Drillich. 2014. Farm characteristics and calf management practices on dairy farms with and without diarrhea: A case-control study to investigate risk factors for calf diarrhea. J. Dairy Sci. 97:5110-5119.

Moon, H. W., and T. O. Bunn. 1993. Vaccines for preventing enterotoxigenic Escherichia coli infections in farm animals. Vaccine 11:213-220.

Moon, H. W., A. W. McClurkin, R. E. Isaacson, J. Pohlenz, S. M. Skartvedt, K. G. Gillette, and A. L. Baetz. 1978. Pathogenic relationships of rotavirus, Escherichia coli, and other agents in mixed infections in calves. J. Am. Vet. Med. Assoc. 173:577-583.

Muscato, T. V., L. O. Tedeschi, and J. B. Russell. 2002. The effect of ruminal fluid preparations on the growth and health of newborn, milk-fed dairy calves. J. Dairy Sci. 85:648-656.

Nagy, B. 1980. Vaccination of cows with a K99 extract to protect newborn calves against experimental enterotoxic colibacillosis. Infect. Immun. 27:21-24.
Nagy, B., and P. Z. Fekete. 2005. Enterotoxigenic Escherichia coli in veterinary medicine. Int. J. Med. Microbiol. 295:443-454.

Naylor, J. M. 2009. Neonatal calf diarrhea. Pages 70-77 in Food Animal Practice. 5th ed. D. E. Anderson and D. M. Rings, ed. W.B. Saunders, St. Louis, MO.

O'Handley, R., C. Cockwill, T. A. McAllister, M. Jelinski, D. W Morck, and M. E. Olson. 1999. Duration of naturally acquired giardiosis and cryptosporidiosis in dairy calves and their association with diarrhea. J. Am. Vet. Med. Assoc. 214:391-396.

Phillips, R. W., L. D. Lewis, and K. L. Knox. 1971. Alterations in body water turnover and distribution in neonatal calves with acute diarrhea. Ann. N. Y. Acad. Sci. 176:231-243.

Radostits, O. M. 1975. Treatment and control of neonatal diarrhea in calves. J. Dairy Sci. 58:464-470.

Sunderland, S. J., P. Sarasola, T. G. Rowan, C. J. Giles, and D. G. Smith. 2003. Efficacy of danofloxacin 18\% injectable solution in the treatment of Escherichia coli diarrhoea in young calves in Europe. Res. Vet. Sci. 74:171-178.

Thiagarajah, J. R., and A. Verkman. 2003. CFTR pharmacology and its role in intestinal fluid secretion. Curr. Opin. Pharmacol. 3:594599

Thiagarajah, J. R., and A. Verkman. 2013. Chloride channel-targeted therapy for secretory diarrheas. Curr. Opin. Pharmacol. 13:888894.

Thorns, C. J., J. A. A. Sawtell, J. A. Miller, and G. W. Wood. 1990. Identification of the K99 (F5) fimbrial adhesin in commercial vaccines used against calf enteritis. Biologicals 18:113-115.

Tradtrantip, L., W. Namkung, and A. S. Verkman. 2010. Crofelemer, an antisecretory antidiarrheal proanthocyanidin oligomer extracted from Croton lechleri, targets two distinct intestinal chloride channels. Mol. Pharmacol. 77:69-78.

USDA. 2011. Dairy heifer raiser: An overview of operations that specialize in raising dairy heifers. National Animal Health Monitoring System, USDA, Fort Collins, CO. http://nahms.aphis.usda.gov.

Walker, P. G., P. D. Constable, D. E. Morin, J. K. Drackley, J. H. Foreman, and J. C. Thurmon. 1998. A reliable, practical, and economical protocol for inducing diarrhea and severe dehydration in the neonatal calf. Can. J. Vet. Res. 62:205-213.

Younis, E. E., A. M. Ahmed, S. A. El-Khodery, S. A. Osman, and Y. F. I. El-Naker. 2009. Molecular screening and risk factors of enterotoxigenic Escherichia coli and Salmonella spp. in diarrheic neonatal calves in egypt. Res. Vet. Sci. 87:373-379. 